\title{
Borderline Ovarian Serous Tumor/Atypical Proliferative Ovarian Serous Tumor
}

National Cancer Institute

\section{Source}

National Cancer Institute. Borderline Ovarian Serous Tumor/Atypical Proliferative Ovarian Serous Tumor. NCI Thesaurus. Code C122584.

A non-invasive serous neoplasm that arises from the ovary and shows greater cellular proliferation and cytologic atypia as compared to benign ovarian serous tumors, but less as compared to low-grade ovarian serous carcinoma. 